\title{
Entre unité et diversité
}

L'histoire et son enseignement en Fédération de Russie

Between unity and diversity. History and how it is taught in the Russian

Federation

Entre unidad y diversidad. La enseñanza de la historia en la Federación Rusa

\section{Aleksei Kilin}

Traducteur : François-Xavier Nérard

\section{OpenEdition}

\section{Journals}

Édition électronique

URL : https://journals.openedition.org/ries/4427

DOI : $10.4000 /$ ries.4427

ISSN : 2261-4265

\section{Éditeur}

France Education international

\section{Édition imprimée}

Date de publication : 1 septembre 2015

Pagination : 65-76

ISSN : $1254-4590$

\section{Référence électronique}

Aleksei Kilin, «Entre unité et diversité », Revue internationale d'éducation de Sèvres [En ligne], 69 |

septembre 2015, mis en ligne le 01 septembre 2017, consulté le 02 juillet 2021. URL : http:// journals.openedition.org/ries/4427 ; DOI : https://doi.org/10.4000/ries.4427 


\title{
Entre unité et diversité
}

\section{L'histoire et son enseignement en Fédération de Russie*}

\author{
Aleksei Kilin \\ Université de l'Oural, Russie
}

«Connaître le passé, comprendre le présent et prévoir le futur »: dans ce contexte, l'histoire prend une actualité et une signification particulières, aussi bien pour comprendre la place de la Russie dans le monde contemporain, que pour permettre à chaque individu de trouver sa place dans le monde. Si l'on ne considère pas seulement l'histoire comme la "science du passé » mais bien comme un processus d'éducation de l'individu "dans le temps et dans l'espace », nous élargissons son horizon. Cela lui donne une importante dimension humaniste, un rôle majeur dans la formation de la vision du monde des individus. Cette conception de l'histoire la fait néanmoins entrer dans le champ des luttes idéologiques, elle est partie prenante de campagnes de propagande et des guerres de l'information, elle fait l'objet d'une attention aiguë de la part des hommes politiques.

Si l'on demande à des passants dans la rue ce qu'est "l'histoire », on ne recevra pas des réponses homogènes. La plupart des personnes interrogées se rappellent des cours suivis à l'école. Certains diront qu'il s'agit de récits du passé, de la mémoire de leurs ancêtres, de leur famille. D’autres évoqueront peut-être des dates de commémorations ou d'événements du passé qui ont marqué le destin du peuple, source de fierté pour le pays. Il est plus rare que l'on mentionne l'histoire en tant que science.

Cet article analyse l'enseignement de l'histoire dans les établissements d'enseignement secondaire et supérieur de Russie et les influences qu'il peut subir.

\section{L'ENSEIGNEMENT DE L'HISTOIRE À PARTIR DE 1992}

Les rapports du nouvel État russe à l'enseignement de l'histoire ont connu deux phases principales. Si, au cours de la première d'entre elles (les années 1990), l'enjeu principal consistait en la création d'un " nouveau » système, chargé de remplacer la conception marxiste-léniniste et de permettre l'existence d'une multitude d'approches différentes, la seconde (les années 2000) repose sur une évidente critique de la première et témoigne d'une tendance à l'unification et à l'utilisation de "rétro-innovations » empruntées au passé prérévolutionnaire et soviétique.

\footnotetext{
* Article traduit par François-Xavier Nérard.
} 
La loi «Sur l'éducation» (1992) énonçait les principes de la politique nationale d'éducation, et parmi eux « le caractère démocratique, social et étatique de la gestion de l'éducation », ce qui sous-entendait le renoncement par l'État au monopole de décision sur toutes les questions de principe liées au fonctionnement et au développement du système éducatif. Elle supposait donc la mise en place d'une collaboration entre l'État et la société dans l'élaboration et la réalisation de la politique nationale d'éducation.

L'histoire de la Russie a toujours été une histoire de l'État russe, de ses dirigeants et de ses institutions. Bien entendu, le changement de système politique et des mécanismes de l'économie qui a eu lieu au tournant des années 1980-1990 avait besoin d'une légitimation. Il est de fait impossible de parler d'un changement radical des élites, puisque que la nomenklatura du parti et de l'État est restée en place, privatisant la propriété publique à son profit et conservant les principales positions de direction du pays. Remarquons que, à la différence des pays d'Europe centrale et orientale, il n'y a eu aucun mécanisme de lustration en Russie. Boris Eltsine, malgré son image de démocrate et de premier président de la Russie, était un ancien membre suppléant du Politburo du Comité central du Parti communiste de l'Union soviétique (PCUS).

Logiquement, dans les recherches historiques, on a assisté à un «changement d'orientation ", à l'abandon du matérialisme historique, et à la mobilisation de nouvelles approches en termes de modernisation, de civilisation, d'anthropologie, etc. On a utilisé beaucoup plus régulièrement des méthodes comparatives, des modélisations mathématiques, des approches pluridisciplinaires, etc.

Avec l'ouverture des archives et le renforcement de la coopération internationale, de nouvelles sources ont été utilisées, et la palette des thèmes scientifiques et de recherche est devenue plus riche et plus diverse. Il ne faudrait pas pourtant surestimer la radicalité des changements. La majorité des historiens, formés dans la tradition marxiste, l'ont en effet perpétuée en y apportant seulement quelques correctifs ici ou là. La composition des manuels d'histoire pour le secondaire et l'université ont logiquement reflété ces changements. Cela s'est traduit par une vaste diversité de manuels qui ont donné aux enseignants et aux élèves la possibilité du choix.

Dans les années 1990, on multiplia les essais pour faire de la place dans les manuels aux différentes interprétations, pour proposer des lectures contradictoires des principaux événements. Face à cette accumulation de faits, de noms et de dates, les élèves perçurent alors l'histoire comme une avalanche étouffante d'informations. Assez logiquement, la réaction fut celle du rejet. C'est pourquoi ces manuels ne jouèrent pas véritablement leur rôle. Les élèves n'essayaient pas véritablement de se forger une idée, mais cherchaient surtout ce qui était la "vérité ». La méthode de l'analyse comparative n'était pas adaptée au niveau du secondaire. 
Cette diversité, comme le souligne l'académicien Andrei Zalizniak (un célèbre linguiste, spécialiste du dialecte de la Novgorod médiévale), était une réaction au dogmatisme dur de la période précédente. Dans cette même logique, certains scientifiques ont cherché à réviser les bases de la connaissance jusqu'à la formulation de théories très douteuses. Leur explosion s'explique par "l'accumulation invisible d'une protestation pendant des décennies de contrôle total de l'idéologie, qui excluait toute alternative au point de vue officiel. Quand le barrage a cédé, la défiance et le désir de verbaliser une opinion alternative sur tout sujet, parfois même adopter tout simplement le point de vue le plus opposé possible, est devenu un phénomène de masse. Les effets positifs étaient là, mais c'était parfois bien stérile. »(Malianov, 2015)

En 1994, le ministère de l'éducation a adopté une "stratégie de développement de l'enseignement de l'histoire et des sciences sociales dans les établissements d'éducation ». Cette stratégie affirmait «le refus du monopole de l'idéologie par l'État, le recours à un système de valeurs, lié aux meilleures traditions nationales et à la tradition universelle de l'humanisme ». (Vjazemskij, Tjuljaeva, 1998)

En août 2001, la question du contenu des manuels scolaires fut examinée lors d'un conseil des ministres du gouvernement de la Fédération de Russie. Puis, en décembre 2003, lors d'une rencontre avec des chercheurs, V. V. Poutine fit la remarque suivante : "Bien sûr, ce n'est pas une mauvaise chose qu'une littérature diversifiée de ce genre se soit développée chez nous..., mais les auteurs aujourd'hui tombent dans l'excès inverse et ignorent le thème du patriotisme, au lieu de développer un sentiment de fierté chez les élèves. »(Sveshnikov, 2004)

En 2013, V. V. Poutine a de nouveau abordé le thème des manuels scolaires : "Il faut réfléchir à la création de manuels uniques d'histoire de la Russie pour l'école secondaire, adaptés aux différents âges.»

\section{L'INTRODUCTION DU « STANDARD HISTORIQUE ET CULTUREL ॥}

En 2014, un «Standard historique et culturel» (guide programmatique) a été adopté et, en avril 2015, le gouvernement a choisi l'ensemble des manuels avec lesquels les élèves russes étudieront à compter du $1^{\mathrm{er}}$ septembre 2015. Les auteurs du Standard sont des chercheurs russes de l'Académie des sciences de Russie, sous la direction du directeur de l'Institut d'histoire universelle, le docteur ès-lettres et académicien A. Tchoubarian ainsi que de deux associations : la Société historique russe et la Société d'histoire militaire.

Le Standard se fonde sur une approche culturelle et anthropologique, à l'opposé d'une histoire " politique ». "Dans les manuels d'histoire contemporains, l'orientation traditionnelle vers l'histoire politique continue à dominer. Elle puise ses racines dans l'école des époques impériale et soviétique. Dans ce 
Standard historique et culturel, une large place est faite à l'individu dans l'histoire, non seulement à travers l'étude des biographies de personnages célèbres, mais également par le biais des péripéties des "simples citoyens". Leur destin permet d'aborder certains processus sociaux et politiques. ${ }^{1}$

On peut décrire la Fédération de Russie comme un "pays de régions » dont l'histoire est très diverse. Dans la loi «Sur l'éducation » (1992), une place était faite à la dimension régionale aux côtés de la dimension fédérale. Selon Isabelle Ogonowskaya (2013a), auteur de manuels pour l'école secondaire et enseignante dans une classe préparatoire à l'Université : "en prenant connaissance de l'histoire, de la géographie, de la littérature de leur région, les écoliers prennent conscience d'une façon ou d'une autre de leur responsabilité pour le présent et le futur de la région dans laquelle ils vivent. »

Au début des années 2000, la dimension régionale s'est progressivement réduite, mais dans le Standard historique et culturel, on affirme de nouveau la nécessité d'une dimension régionale et ethnoculturelle à travers la formulation suivante: «l'histoire du pays à travers l'histoire des régions".

Remarquons que dans les établissements d'enseignement supérieur, la dimension régionale persiste. Cela s'explique par le fait que la majorité des historiens, travaillant sur l'histoire nationale, utilisent activement des matériaux régionaux et étudient les spécificités régionales en s’appuyant sur les archives locales.

Un autre problème de l'enseignement de l'histoire à l'école est celui de l'interaction de cette discipline avec les autres matières comme la littérature, les sciences sociales ou la géographie. D’après I. Ogonowskaya (2012), " la plupart des exercices, qui mettent en jeu les valeurs des élèves, sont proposés au cours des leçons de disciplines littéraires. Le contenu et la structure des programmes de ces matières, comme la littérature, l'histoire, les sciences sociales, l'économie, le droit, la culture mondiale et en partie, la géographie, ne sont absolument pas coordonnés et toujours pas intégrés. Chacune de ces disciplines vit de façon autonome dans le champ des humanités. »

Cela s'explique essentiellement, selon les professeurs, par l'autonomie et le manque de coordination des programmes par discipline.

L'aggravation de la situation politique, la mobilisation des technologies de propagande, ont pour conséquence inévitable l'utilisation d'une grande quantité d'informations historiques dans le contexte politique actuel. Ces informations sont mises au goût du jour, en partie modernisées, adaptées en fonction de la conjoncture et des goûts du public. La "popularisation» de l'histoire devient une vulgarisation et la multiplication des stéréotypes favorise les représentations mythiques du passé. L'influence des technologies médiatiques, mobilisées en fonction de la pensée « clipesque » de la jeunesse, ne peut pas être comparée avec l'influence potentielle d'un manuel scolaire. Dans l'espace médiatique actuel,

1. Toutes les références de cet article au Standard proviennent du dossier du ministère de l'éducation et de la science de la Fédération de Russie [en ligne] : [http://goo.gl/2kco6m]. 
l'image domine le texte. Un exemple frappant est le résultat du concours «le nom de la Russie, choix historique 2008 » remporté, selon le professeur Igor Danilevski, par l'acteur Nikolai Tcherkassov dans le rôle du prince Alexandre Nevski, alors qu'on ne sait - non seulement les citoyens, mais également les historiens professionnels - que très peu de choses sur le prince de Novgorod.

En prenant cela en considération, les auteurs du Standard proposent de considérer les manuels scolaires comme un navigateur, une sorte d'intégrateur de ressources informationnelles. "Dans des conditions de développement des moyens de communication et de l'accès dans l'immense majorité des écoles à l'Internet haut débit, le rôle du manuel comme conservateur du savoir prend de nouveaux traits et particularités. Le manuel doit non seulement donner des informations et proposer des interprétations, mais inciter les élèves à penser par eux-mêmes, à analyser les textes historiques, à tirer des conclusions, etc. »

La période soviétique de l'histoire de la Russie a apporté d'importants correctifs aux valeurs familiales. Les répressions de masse ont parfois contraint les individus à " oublier » leur passé, à prendre de la distance ou à totalement rompre avec leurs ancêtres. Tout le monde n'avait pas des commandants rouges, des commissaires ou des bolcheviks dans sa famille, nombreux étaient ceux qui avaient des ancêtres paysans, souvent dékoulakisés et parfois même condamnés. L'histoire de Pavlik Morozov, quand un fils dénonce son père, était un exemple dans la période soviétique. Dans les années 1990 a eu lieu un important déplacement des valeurs et ce qui, auparavant, était tu, est passé au premier plan. La mémoire historique a été progressivement restaurée, ou en tout cas reconstruite.

D’un certain côté, il y avait une volonté évidente de comprendre le passé et de l'autre un manque de forces et de temps, car dans le contexte de réformes permanentes de cette époque, les gens essayaient surtout de s'adapter et de survivre. L'indétermination du futur a réduit proportionnellement la profondeur de l'implication dans le passé. Le manque de repères solides a conduit les citoyens à s'appuyer sur les "valeurs traditionnelles" notamment religieuses et ethniques (dans une interprétation approximative, populaire et parfois biaisée ou radicale). La vie dans des circonstances extrêmes ne donne pas la possibilité de prendre du recul par rapport à la routine quotidienne, aux nécessités du quotidien pour essayer de se projeter dans un contexte historique plus vaste. Elle prive ainsi de mémoire historique. C'est clairement une situation contradictoire et conflictuelle qui est source de stress pour tout individu et contreproductive pour le système d'enseignement. C'est l'une des raisons pour lesquelles le rôle des associations locales dans la formation et la conservation de la mémoire historique est faible. Les germes de la société civile n'ont pas le temps de pousser : le pouvoir y voit souvent une menace. On peut considérer positivement la mention dans le Standard historico-culturel de "l'activité civique » avec quelques nuances toutefois : " [...], ceci dit, il faut dresser une ligne claire entre les "manifestations normales" de l'activité civique et toutes sortes d'extrémismes, de terrorismes, de chauvinismes, de propagandes de l'exception nationale, etc. » 
La tendance principale qui domine aussi bien la formation des valeurs au niveau de la propagande d'État que dans les programmes d'éducation est le " conservatisme ». Le recours au passé, aux valeurs traditionnelles, qui sont censées être opposées aux valeurs des démocraties occidentales, fait des liens de l'État avec la religion un lien organique. L'introduction dans les programmes du secondaire d'un cours consacré aux "Bases de la culture religieuse et de l'éthique laïque " a eu un vaste écho dans la société russe. Si, théoriquement, ce cours correspond à la législation nationale, on constate dans la pratique de nombreuses contradictions, par exemple la segmentation des élèves en fonction des convictions religieuses. La thèse du rôle particulier de l'orthodoxie dans la culture nationale a été incluse dans le texte du Standard historique et culturel : «L'histoire des religions, en premier lieu de l'orthodoxie, doit être présentée systématiquement et pénétrer tout le contenu du manuel. Il est obligatoire également d'inclure dans le manuel des données sur la diffusion des principales confessions non-chrétiennes (islam, judaïsme et bouddhisme) sur le territoire russe. »

\section{UNE BIPOLARITÉ TRADITION NELLE}

La tradition historique nationale s'est toujours construite sur un dualisme "Ouest-Est», sur leur opposition et leur concurrence. Une certaine conception tente d'inscrire l'histoire de la Russie dans un contexte mondial, tandis que l'autre insiste sur son caractère exceptionnel. Cette lutte à travers les siècles a pris les formes les plus diverses : normanistes et antinormanistes, occidentalistes et slavophiles, partisans de la révolution mondiale et ceux de la construction du socialisme dans un seul pays, libéraux et patriotes. La dialectique du processus est très productive tant que l'une des deux tendances ne prend pas le pouvoir d'État et ne forme pas son idéologie.

Un rôle important dans ce contexte est joué par l'espace. La géopolitique, avec le rôle fondamental qu'elle accorde au facteur géographique, passe ces derniers temps au premier plan. La taille de l'Empire russe, de l'URSS puis de la Fédération de Russie (même si elle est plus réduite) est un argument de poids pour une approche impériale. "La Russie était, est et continuera d'être un empire » : cette thèse, toute archaïque qu'elle soit, est partagée par de vastes couches de la population et par les élites au pouvoir. C'est un élément de la mythologie nationale et non un fait pensé rationnellement, vu que la conception de l'empire ne correspond ni à la construction fédérale de la Russie, ni aux principes normatifs de sa gestion, ni à son modèle économique.

La grandeur de l'espace se projette sur la grandeur de la nation, et plus précisément de son État. «La Russie est le plus grand pays du monde. En fonction de cela, une conscience historique nationale s'est formée: nous sommes les citoyens d'un grand pays avec un grand passé. » 
L'absence de résultats significatifs dans la sphère politique et économique, l'orientation dans la sphère idéologique vers des images et des symboles du passé, imposent d'avoir recours au patriotisme, qui est pensé comme une forme de fierté du pays. Par " pays », on comprend, avant tout, l'État, indépendamment de ce qu'a fait cet État ou des conséquences de ses actions. Sur ce fond, on comprend mieux les essais destinés à réhabiliter la période stalinienne, à présenter I. Staline comme un manager efficace, capable de résoudre des problèmes de gestion très complexes.

Dans les travaux contemporains de vulgarisation historique, comme dans les études à la fois fondamentales et appliquées, on constate une tendance à une certaine prise de conscience nationale, glorifiant une certaine grandeur, censée compenser les dommages subis par l'URSS qui a perdu la course aux armements et la compétition économique avec les États-Unis. La chute de l'URSS aujourd'hui est présentée comme une tragédie, et les formes d'intégration dans le cadre de la CEI, de l'Union douanière ou du « monde russe » comme un moyen de la dépasser.

La formation du patriotisme est considérée par beaucoup d'enseignants comme la conséquence d'une approche dogmatique de tel ou tel sujet de l'histoire nationale (principalement positifs). Du point de vue de l'État, qui considère que les citoyens sont l'objet de son action, c'est tout à fait justifié. L'argument de base est le suivant: l'idéologie de l'État doit être appliquée dans les écoles financées avec l'argent de l'État. On peut pourtant penser que les impôts, que payent les parents, accumulés et distribués par l'État, doivent être dépensés sur des projets que les citoyens estiment les plus significatifs, comme la formation d'un esprit créatif et critique, la capacité de s'orienter dans les différentes situations de la vie, dans n'importe quel endroit du globe. Bien entendu, cette conception ne peut fonctionner qu'avec la création d'un système transparent et efficace de représentation populaire.

Parler du renforcement de la cohésion de la société russe contemporaine est très complexe, vu que les citoyens se trouvent dans des champs informatifs et idéologiques différents. La société est plus divisée que jamais ; même s’il s'agit de groupes de taille très différente, ils restent significatifs. Il existe de moins en moins d'espaces de dialogue. Les efforts d'homogénéisation de la population passent non pas par la recherche d'un compromis entre les différentes positions, mais par l'exclusion des opposants politiques de l'espace légal. C'est bien pourquoi les rangs des "agents de l'étranger» se font plus nombreux, notamment grâce à des organisations sociales qui prônent la conservation de la mémoire historique. C'est le cas par exemple de l'association Mémorial.

La consolidation de la société russe ne s'est jamais faite au moyen de facteurs positifs, internes, mais contre les autres, les étrangers : la menace extérieure, celle de désintégration, de schisme, le séparatisme, le localisme, des influences culturelles extérieures, la propagande de l'autre, l'opportunisme, « la tolérance asexuée », les « agents de l'étranger » ou les « ennemis du peuple ». 
Le paradoxe de la situation tient à ce que pour entretenir un certain degré de tension, il faut sans cesse jeter au feu de nouveaux " ennemis » et en trouver d'autres quand ces ennemis s'épuisent.

Dans les conditions d'indétermination idéologique, on essaie de conjuguer "tout le meilleur », notamment les attributs et les valeurs tirés du passé impérial et soviétique. Le syncrétisme est évident dans les symboles de l'État: gerbe impériale, drapeau bourgeois, hymne soviétique.

La liste des dates des commémorations officielles souligne bien ce phénomène. En plus de Noël, on fête la Journée internationale de la femme le 8 mars. Dans ce kaléidoscope des célébrations, il y a quelques points de base, des repères qui rassemblent la société et vis-à-vis desquels il existe un consensus. Outre le Nouvel An, c'est le cas du Jour de la Victoire. La période de la Grande Guerre patriotique de 1941-45 n'est pas si éloignée, c’est pourquoi le sens de la victoire est ressenti aussi nettement par tous les citoyens. L'idée selon laquelle on est prêt à tout pour ne pas "revivre la guerre » revient sans cesse dans la bouche des générations plus âgées. C'est la guerre qui unit la société, c'est pourquoi l'image de l'ennemi extérieur et les analogies avec cette période servent de puissants moyens de mobilisation, même dans les circonstances contemporaines, 70 ans plus tard. Malheureusement, ce capital historique de grande valeur est utilisé dans des campagnes de propagande qui le dévalorisent et détruisent un consensus national déjà bien fragile sans cela. "Les relations de la Russie avec les autres États et les autres peuples sont le plus souvent étudiées sous le prisme des guerres, des conquêtes, des conflits et non à travers les interactions, les influences croisées, l'enrichissement des cultures. (I. Ogonowskaya, 2012)»

La démilitarisation des consciences est l'un des buts poursuivis par les auteurs du Standard historique et culturel lorsqu'ils insistent sur différents aspects de l'histoire des guerres. «Il ne faut pas que seules les victoires militaires créent un sentiment positif de la conscience historique, l'attention la plus vive doit être portée aux réussites du pays dans d'autres domaines. Doivent faire également l'objet de la fierté patriotique l'immense effort du peuple pour la conquête des vastes espaces de l'Eurasie avec sa nature difficile, la formation de la société russe dans sa dimension multinationale et multiconfessionnelle, dans le cadre duquel dominaient l'entraide, la tolérance, la création de science et de culture de niveau mondial. »

C'est pourtant bien les guerres qui sont le moyen grâce auquel l'élève d'aujourd'hui prend connaissance de l'histoire des pays étrangers. Le professeur Vladimir Zemtsov (spécialiste de la France à l'époque des guerres napoléoniennes) note ainsi : "L'introduction de l'examen unique d'état (EUE-EGE) a rendu pour beaucoup inutile l'enseignement de l'histoire des pays étrangers, elle a rendu nulle la motivation pour l'étude de l'histoire du monde.»

La réduction du volume horaire et l'orientation vers l'EUE conduit à la répartition des horaires en faveur de l'histoire nationale, en réduisant le champ 
de vision de l'élève. Cela optimise certes la charge de travail, mais réduit la vision du monde de l'individu, cela le rend moins sensible à l'autre et renforce la démarche de confrontation.

Dans le contexte géopolitique contemporain, la Russie s'oppose au monde extérieur. L'aggravation de la situation en politique extérieure permet de consolider la société autour de l'image de l'ennemi. C'est particulièrement le cas de conflits non seulement avec nos ennemis lointains traditionnels de la période de la guerre froide, mais aussi avec nos proches voisins, la Géorgie, la Moldavie, l'Ukraine, avec les peuples de pays qui étaient traditionnellement perçus comme des amis par la majorité des Russes. Il n'y a pas eu de peuple plus proche des Russes que les Ukrainiens pendant longtemps. Pour les politologues, la dernière frontière est le rapport aux Tatares, qui sont les plus intégrés à la réalité russe. Si un conflit apparaît ici, on pourra s'interroger sur l'unité de l'État.

Les auteurs du Standard, en essayant de rompre avec cette tendance négative, essaient de changer l'évaluation de la politique coloniale russe. "Un moment essentiel de cette conception doit être la compréhension du passé de la Russie comme une partie inaliénable d'un processus historique mondial. Il est essentiel d'élargir le volume des enseignements sur l'histoire des peuples de la Russie, en mettant l'accent sur les interactions des cultures, sur le renforcement des liens économiques, sociaux, politiques et autres entre les peuples. Il s'agit d'évoquer l'histoire des relations entre nationalités dans toutes les étapes de l'histoire nationale. Il faut souligner le fait que l'inclusion dans l'Empire russe a eu des aspects positifs pour ces peuples : sécurité contre les ennemis extérieurs, fin des troubles intérieurs et des guerres civiles, développement économique, diffusion des lumières, éducation, santé, etc. »

En contrepoids à «l'analyse multifactorielle » et dans la logique des stéréotypes de la conscience de masse, la théorie du complot est très populaire en Russie, répandue par les médias et donne une interprétation simple et univoque de tous les échecs et défaites. Elle s'inscrit symboliquement dans le modèle de la "pensée de la confrontation ». Cette théorie, au minimum, est méprisante, car elle fait totalement reposer la responsabilité de nos actions sur d'autres, nous refusant le rôle d'acteur de l'histoire. La popularité de cette idée a contraint les auteurs du Standard à y accorder leur attention : " Il faut créer une représentation claire chez les écoliers : les révolutions et les guerres civiles ne sont pas le résultat d'un complot intérieur ou extérieur, mais la conséquence de contradictions objectives à l'intérieur du pays. »

\section{Des influences MULTIPLES}

Le système d'éducation en général et l'éducation historique en particulier sont soumis aujourd'hui à l'influence de plusieurs facteurs. C'est la situation économique du pays, la situation de la Russie dans l'arène internationale et la recherche d'une idéologie d'État sous la forme d'une "idée russe ", qui aiderait à la consolidation comme de l'État et de la société. 
Le système éducatif en Russie, formé dans la période précédente, ne correspondait pas au niveau de développement de l'économie et du système politique. Dans les conditions de la «stabilité » et de la "stagnation », il était nécessaire de conserver la structure sociale. Mais dans le cadre d'une économie de matières premières, totalement incluse au sein d'un système industriel, il faut se séparer de tous ceux qui n'entrent pas dans ce cadre. D’où le but formulé de l'éducation : former non pas un "citoyen » mais un "travailleur éduqué » ou (dans la version de l'ancien ministre de l'éducation et actuel conseiller du président de la Fédération de Russie, Andrei Foursenko) un "consommateur éduqué ».

Le statut de grande puissance suppose la manifestation du leadership non seulement dans le champ militaire mais aussi dans le domaine de l'éducation. Le gouvernement a ainsi mis en place le projet « 5-100» dont le but est la maximisation de la position concurrentielle des plus grandes universités russes sur le marché global des services éducatifs et des programmes de recherche. Dans le cadre de ce projet, cinq principales universités russes doivent entrer dans les cent plus grands établissements mondiaux ${ }^{2}$.

I. Ogonowskaya (2013c), en analysant les problèmes de l'école contemporaine, relève la bureaucratisation, qui se traduit un style autocratique de gestion et la formalisation du processus d'éducation. "Les fonctionnaires et l'école se sont mis à s'adapter à l'instrument EUE : orienter la formation dans la logique de la "préparation" à l'examen sous la forme d'exercices standardisés au format de l'EUE, organiser sous ce format des olympiades à différents niveaux... Le système éducatif lui-même a commencé à se dégrader. [...] La présentation formalisée des résultats de la formation a engendré un rapport formalisé au processus éducatif lui-même. Ceci dit, des élèves pensant de façon originale ne sont pas moins efficaces aux examens du format ECE. »

L'accent mis sur la préparation à l'EUE, en fait, a fait disparaître la question de la diversité des programmes d'enseignement car le processus d'enseignement est désormais déterminé non pas par le manuel choisi, le niveau de qualification ou la position citoyenne du professeur, mais par les questions de l'EUE. La préparation au test et les bilans, qui sont les principaux critères d'évaluation de l'activité du professeur, éliminent de l'école des méthodes éducatives orientées sur l'acquisition de pratiques de communication, d'une réflexion critique, d'une logique de pensée. Les questions supposant l'examen de points de vue opposés (dans la partie C de l'EUE) ne compensent pas les défauts mentionnés.

Le système d'éducation aujourd'hui est l'objet de nombreuses expérimentations, le lieu où se réalisent les idées des théoriciens de l'éducation et de fonctionnaires. Selon plusieurs blogueurs, ces " anciens cancres se vengent de l'école pour leurs anciens échecs ". C'est une explication ironique, mais elle reflète la position d'une partie de la société face à ce qui se passe.

2. Voir la bibliographie en fin d'article. 
L'éducation supérieure et secondaire, notamment l'enseignement de l'histoire, est liée de façon indissociable et interdépendante; plongée dans un processus permanent de changement, elle est contrainte de répondre efficacement aux demandes intérieures et extérieures, non seulement pour se développer, mais également pour se protéger. Ces circonstances font du problème de l'enseignement de l'histoire une question absolument passionnante, parfois dramatique, intrigante au point de ressembler à un roman policier : personne ne peut prédire le dénouement, ni répondre à la question de savoir comment cela va finir.

\section{BIBLIOGRAPHIE}

MALIANOV DA Zaliznyak (2015), "Fomenko - une victime du fanatisme », Gazeta.ru. [http://goo.gl/H1Mfxn], page consultée le 5 avril 2015 [en russe].

OGONOWSKAYA I.S. (2013a) : "L'enseignement de l'histoire dans les écoles russes dans les années 1990-2000: Le champ des réformes sous une perspective juridique ", dans I.S. Ogonovskaya (dir.), L'histoire russe sous l'angle du droit, des destinées humaines et des perspectives réelles: matériaux scientifiques et pratiques, Conférence pédagogique, scientifique et pratique, p. 68-79, Ekaterinburg: éditions Pravovoe Obrazovanie, Éducation juridique - $\mathrm{XXI}^{\mathrm{e}}$ siècle [en russe].

OGONOWSKAYA I.S. (2013b) : "Contexte socio-culturel des standards éducatifs de l'État fédéral : l'environnement de la résolution de problèmes en éducation ", dans : E.M. Dorozhkin, V.A. Fedorov (dir.), Processus d'innovation en éducation: stratégie, théorie et pratiques de développement: Conclusions de la VIe Conférence scientifique et pratique de toutes les Russies, Ekaterinburg, University Press, p. 31-34 [en russe].

OGONOWSKAYA I.S. (2013c) : «L'éducation libérée du bureaucratisme comme facteur de consolidation de la société russe», in Ogonovskaya I.S., Tchernykh N. A. (dir.), Actes de la conférence "L'idée nationale dans la Russie contemporaine ", Ekaterinburg, éditions AMB, 2013, p. 121-142.

OGONOWSKAYA I.S. (2012) : "La ligne ethnoculturelle dans l'enseignement de l'histoire à l'école : contenu et mise en application », in Tchoubarian A. O. (dir.), Actes de la conférence: l'enseignement de la dimension ethnoculturelle dans le cadre de l'histoire de la Russie, Ufa - Université pédagogique d’État de Biélorussie. 19-20 avril 2012, 2012, p. 162-168 [en russe].

SVESHNIKOV A. (2004) : "Tendances de base et résultats ", Neprikosnovennyj zapas, n 4 (36), 2004, p. 70-77. [http://magazines.russ.ru/nz/2004/4/sv10.html], consulté le 2 août 2015 [en russe].

VJAZEMSKIJ E.E., TJULJAEVA T.I. (1998) : Stratégie de développement de l'enseignement d'histoire et de sciences sociales dans les établissements du secondaire, approuvée sur décision du collège du ministère de l'éducation de la Fédération de Russie, 28 décembre 1994, in Memento pour l'enseignant des disciplines de connaissance de la société $\mathrm{n}^{\circ}$ 24/1, Moscou, CGO, p. 26-31 [en russe].

ZEMTSOV V.N. (2010) : "Histoire contemporaine de la Russie dans le contexte de l'histoire mondiale : comment enseigner l'histoire des pays étrangers à l'école russe ", dans L. A. Zaks (dir.), Actes de la conférence: 20 années de Russie post-soviétique: phénomènes de crise et mécanisme de modernisation, Ekaterinburg, Presses de l'Université des Sciences humaines, tome 1, p. 319-323 [en russe]. 


\section{En ligne}

"Sur l'éducation en Fédération de Russie », loi fédérale n 273-FZ du 29 décembre 2012 [http://goo.gl/LnV6h5] [page consultée le 21 avril 2015] [en russe].

Pour en savoir plus sur le débat public sur le projet de Standard historique et culturel, voir le portail officiel du ministère de l'éducation et de la science de la Fédération de Russie : [http://minobrnauki.rf/dokumenty/3483] [en russe].

Sur « 5-100», le projet d'amélioration de la compétitivité des plus grandes universités russes par rapport aux plus grands centres mondiaux d'enseignement et de recherche, voir [http://5top100.ru], page consultée le 18.04.2015 [en russe et en anglais]. 